\title{
A Measurement Based Model for UMTS DL DCH Dynamic Bearer Type Switching
}

\author{
Wolfgang Karner, Olivia Nemethova, Markus Rupp \\ Institute of Communications and Radio-Frequency Engineering \\ Vienna University of Technology, Austria \\ Gusshausstrasse 25/389, A-1040 Vienna, Austria \\ Email: \{wkarner, onemeth, mrupp\}@nt.tuwien.ac.at
}

\begin{abstract}
GPP specifications allow to change the physical resources allocated to the users dynamically to optimize the radio resource utilization in UMTS. Network Operators can make use of this optimization feature by properly adjusting dynamic bearer type switching in their networks. In this paper we show an analysis of the dynamic bearer type switching based on measurements in a live UMTS network in the city center of Vienna, Austria. Particularly, we present the usage probability and the runlength distribution of the three different radio access bearers which are used for transmission over the UMTS DCH (Dedicated Channel) packet switched (PS) domain in DL (Down Link), namely 384kbit/s, 128kbit/s and $64 \mathrm{kbit} / \mathrm{s}$ bearer. For the performance evaluation of applications, system level simulators are used, where the lower layers are usually represented via stochastic models. Following the measured statistics we develop a model capable of properly describing the dynamic switching between the mentioned bearers. Furthermore, we show that a four-state Markov model with its geometrically distributed runlengths does not provide sufficient accuracy. We achieve an enhancement by using a four-state renewal process with Weibull distributed runlengths as presented in this document.
\end{abstract}

\section{INTRODUCTION}

Its high flexibility is one of the main advantages offered by UMTS. This means the data rate in UMTS can be changed every $10 \mathrm{~ms}$, either by changing the transport channel type or by varying the dynamic or semi-static parameters of the transport format (TF)( [1], [2]). Such switching of the channel characteristics optimizes the use of the radio resources and facilitates providing the required quality of service for the user. It can be triggered by admission control, congestion control, soft handover, required throughput or the radio channel quality (power threshold).

In this document we are focusing on the dynamic switching between the three different DCH (Dedicated Channel) DL (Down Link) radio access bearers for the PS (Packet Switched) domain with $64 \mathrm{kbit} / \mathrm{s}, 128 \mathrm{kbit} / \mathrm{s}$ and $384 \mathrm{kbit} / \mathrm{s}$ which are characterized by different values of TTI (Transmission Timing Interval), TBSS (Transport Block Set Size) and SF (Spreading Factor). Our analysis focuses on the dynamic bearer type switching due to fading and coverage reasons, where the decision of up or down switching is based on the link quality only.

For the performance evaluation of applications in system level simulators, models are needed which are capable of representing the error characteristics of the underlying channel properly. In [3] we have developed such a model, assuming absence of dynamic bearer switching. Due to the fact that the error characteristics are different in each bearer a better representation of the channel can be reached by including bearer switching in the error model which is done e.g. in [4] based on simulations only. Our approach in this work is to develop a model for dynamic bearer type switching based on measurements in live UMTS networks within typical mobility scenarios for using UMTS services. This model for dynamic bearer type switching can then be combined with the error model of [3] and the combination can be used as a representation of the lower layers in a system level simulation tool.

This document is organized as follows. In Section II the measurement setup will be explained and the used parameters for traffic and system will be presented. Section III shows the measurement results in detail and in Section IV the model is presented. Finally in Section V a summary and conclusions will be given.

\section{Measurement Setup}

\section{A. General Setup}

All the measurements presented in this document have been realized in a live UMTS network in the city center of Vienna, Austria.

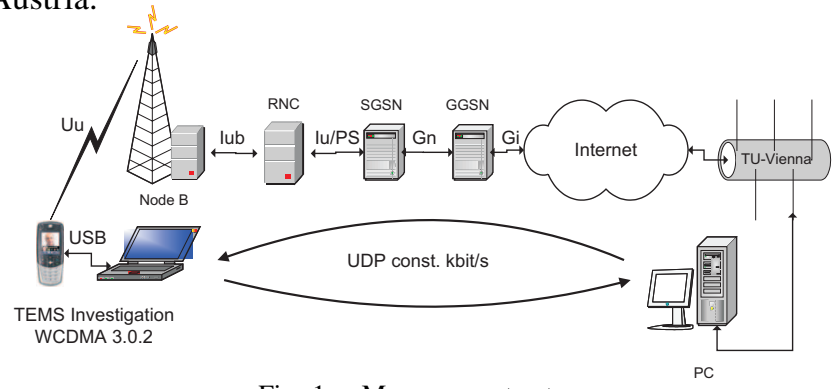

Fig. 1. Measurement setup.

For the measurements a UDP data stream with a bit rate of $360 \mathrm{kbit} / \mathrm{s}$ (372kbit/s incl. UDP/IP overhead) in DL was used. The data was sent from a PC located at the Institute of Communications and Radio-Frequency Engineering at the Vienna University of Technology to a notebook using a UMTS terminal as a modem. As depicted in Fig. 1, the UDP data stream goes from the PC over university LAN (ethernet), Internet, UMTS core network and over the UMTS air interface to 
a UMTS mobile which is connected via USB (Universal Serial Bus) to the notebook. The WCDMA TEMS mobiles ${ }^{1}$ Motorola A835 ('mobile 1') and Sony Ericsson Z1010 ('mobile 2') were both used as terminals. On the notebook the measurements from the mobiles have been captured by 'TEMS Investigation WCDMA 3.0.2' software [5]. By parsing the export files of this software tool, the runlengths (the time to stay at a certain bearer) have been analyzed as explained further on (see Section III) in this document.

As already mentioned, our analysis focuses on the dynamic bearer type switching due to fading and coverage reasons only. Therefore, we assume that during the measurements we had admission for using all the bearers and we also assume a congestion-free network. This means that there were enough resources available in all the cells, so that we could make use of our favourite $(384 \mathrm{kbit} / \mathrm{s})$ bearer. Furthermore, a UDP data traffic with a constant bit rate of $372 \mathrm{kbit} / \mathrm{s}$ should be enough throughput to be assigned a $384 \mathrm{kbit} / \mathrm{s}$ bearer due to throughput constraints.

\section{B. Mobility Scenarios}

For the evaluation of the runlength statistics, several scenarios with different mobility characteristics have been considered which are called: 'static', 'small scale movement', 'walking indoor', 'tram 2', 'car Vienna/Guertel' and 'car highway Vienna-Schwechat'. The measurements for the 'static' case were performed in a room of the Institute of Communications and Radio-Frequency Engineering at the Vienna University of Technology. For these measurements the UMTS terminal was lying on the table in a typical office environment. Due to few movement of persons or other objects around the mobile station, there were only little variations in the channel. The 'small scale movement' measurements were performed by a person sitting at the table and randomly tilting and moving the UMTS mobile with his hands. In the 'walking indoor' scenario, as the label indicates, the measurements were obtained while walking around in the building of the Institute of Communications and Radio Frequency Engineering.

The three scenarios mentioned up to now are indoor scenarios, whereas the following three scenarios are outdoor scenarios measured in the tramway number two going round the city center of Vienna ('tram 2') and going by car either on the street in Vienna called Guertel ('car Vienna/Guertel') with moderate speed or on the highway from Vienna to Schwechat ('car highway Vienna-Schwechat') with higher speeds. The speed distribution during the measurements of the latter two scenarios can be seen in Fig. 2.

\section{Relevant UTRAN Parameters}

In the considered UMTS network, the relevant system parameters are as follows.

As TrCH (Transport Channel) in the DL a DCH (Dedicated Channel) with RLC (Radio Link Control) AM (Acknowledged

\footnotetext{
${ }^{1}$ Modified UMTS mobiles for TEMS data logging, offered by Ericsson [5]. The mobiles are modified in a way that they provide the internal measurements via the interface to the notebook.
}
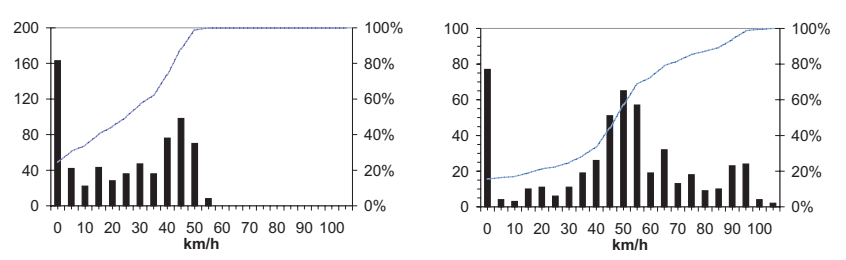

Fig. 2. Histograms and empirical CDFs of speed during measurements driving by car on a) Vienna/Guertel, b) highway Vienna-Schwechat.

Mode) has been used. In addition, the system used turbo coding and a transport block size of 336bits. In case of the $384 \mathrm{kbit} / \mathrm{s}$ bearer the SF (Spreading Factor) was 8 and the TTI (Transmission Timing Interval) was $10 \mathrm{~ms}$ with 12 transport blocks per TTI. The $128 \mathrm{kbit} / \mathrm{s}$ bearer used a SF of 16 , a TTI of $20 \mathrm{~ms}$ with eight transport blocks per TTI and the $64 \mathrm{kbit} / \mathrm{s}$ bearer used a SF of 32, the TTI was $20 \mathrm{~ms}$ and there were four transport blocks transmitted within each TTI. These settings correspond to the RAB (Radio Access Bearer) configurations in [6], [7]. A schematic illustration of the mentioned bearer parameters is shown in Fig. 3.

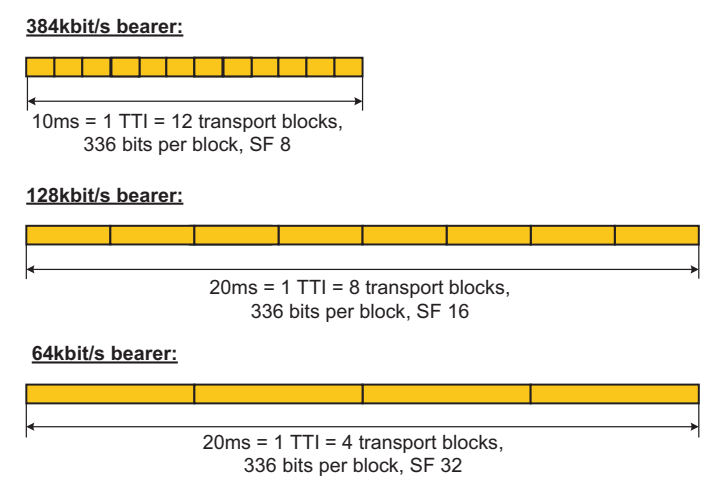

Fig. 3. Illustration of the bearer parameters.

\section{Measurement Results}

In Fig. 4 the usage probability of the various bearers for 'mobile 1 ' is shown (based on the number of TTIs in each bearer). We can observe that in all the scenarios with mobility, the probability of being in the $384 \mathrm{kbit} / \mathrm{s}$ bearer is around $75 \%$ whereas in the static case it is almost $100 \%$. Therefore it is necessary to take dynamic bearer switching into account when modeling the error characteristics for scenarios with mobility.

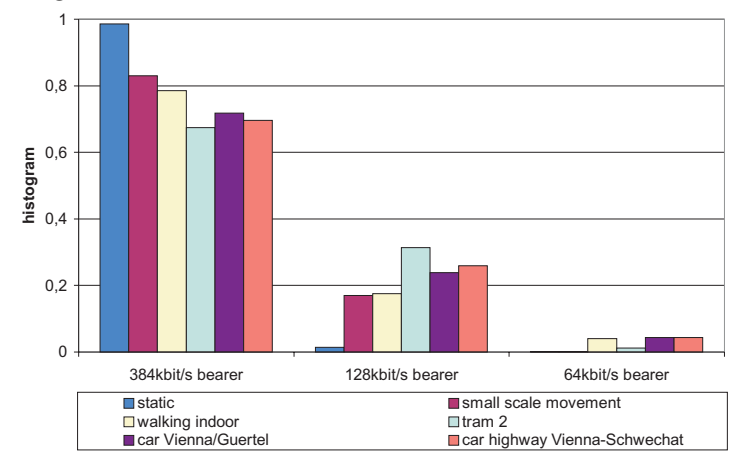

Fig. 4. Usage of 384, 128 and $64 \mathrm{kbit} / \mathrm{s}$ bearer ('mobile 1'). 


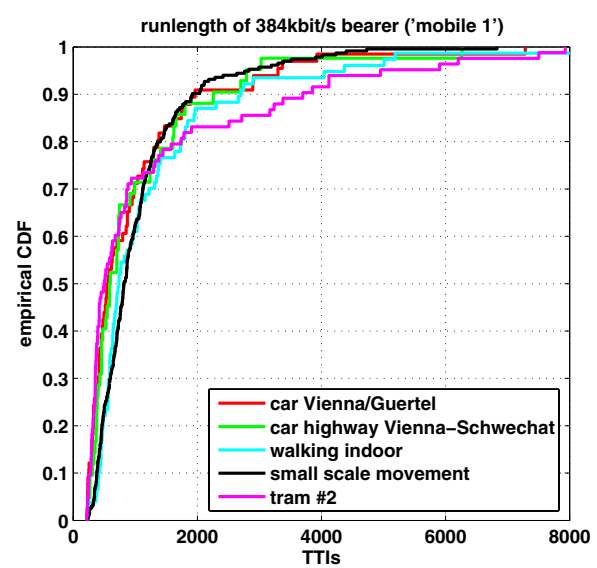

Fig. 5. Measured runlengths of $384 \mathrm{kbit} / \mathrm{s}$ bearer in different scenarios ('mobile 1').

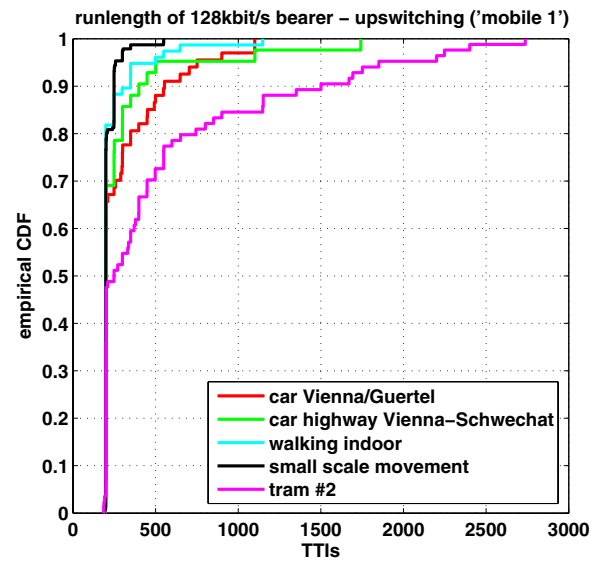

Fig. 6. Measured runlengths of $128 \mathrm{kbit} / \mathrm{s}$ bearer (upswitching) in different scenarios ('mobile 1').

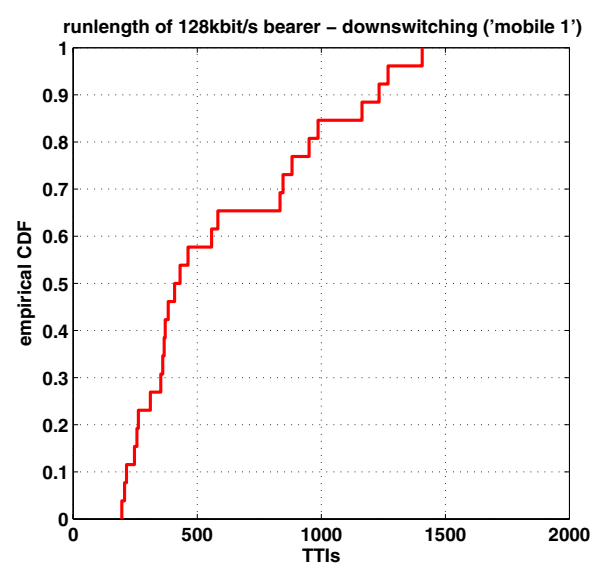

Fig. 7. Measured runlengths of $128 \mathrm{kbit} / \mathrm{s}$ bearer (downswitching), sum of different scenarios ('mobile 1').

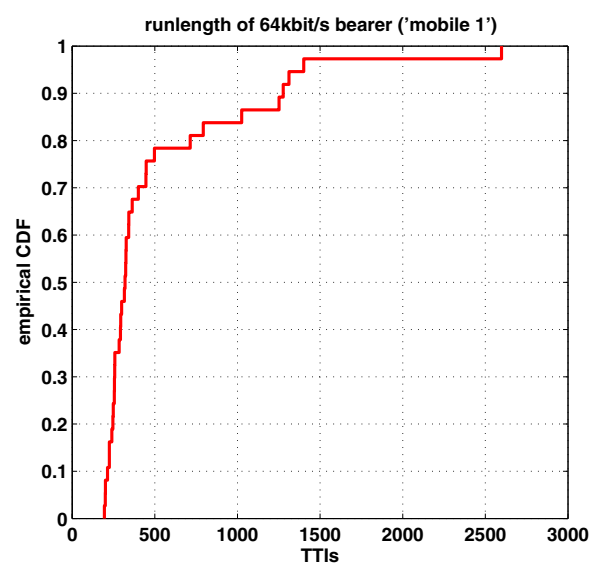

Fig. 8. Measured runlengths of $64 \mathrm{kbit} / \mathrm{s}$ bearer, sum of different scenarios ('mobile 1').

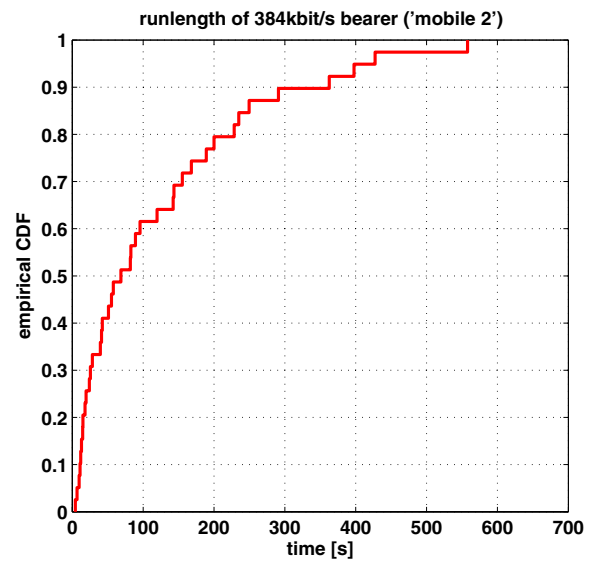

Fig. 9. Measured runlengths of $384 \mathrm{kbit} / \mathrm{s}$ bearer, sum of different scenarios ('mobile 2').

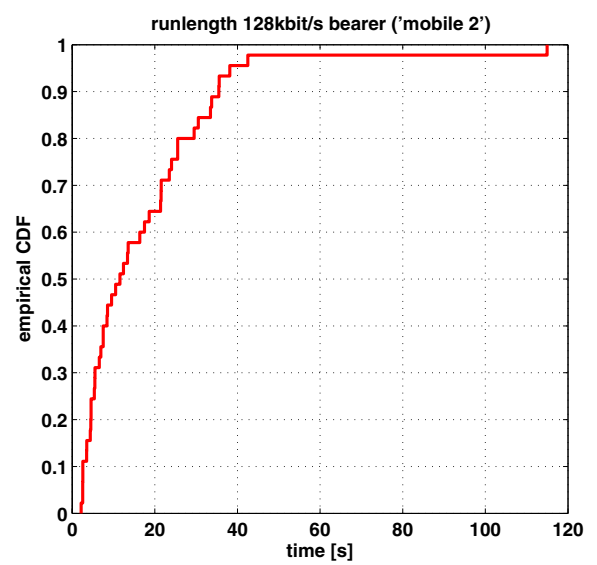

Fig. 10. Measured runlengths of $128 \mathrm{kbit} / \mathrm{s}$ bearer, sum of different scenarios ('mobile 2'). 
Our analysis of dynamic bearer type switching is based on the runlength distribution of each of the bearers, measured in the number of subsequent TTIs in case of 'mobile 1' and in seconds between the 'Transport Channel Reconfiguration' messages for 'mobile 2'. For 'mobile 2' the runlengths are provided in seconds instead of TTIs, due to the fact that with 'mobile 2' it is not possible to analyze the runlengths in the accuracy of TTIs but only by the 'Transport Channel Reconfiguration' messages.

In Fig. 5 the runlengths are shown for the 384kbit/s bearer and various scenarios for 'mobile 1'. We can observe that the minimum runlength is 200 TTIs $(=2 \mathrm{~s}$ in case of $384 \mathrm{kbit} / \mathrm{s}$ bearer) and that $80 \%-90 \%$ of the runlengths are shorter than 2000 TTIs (=20s). Furthermore, we can see that less than $5 \%$ of the runlengths are $>6000$ TTIs (60s) and the longest period to stay in the $384 \mathrm{kbit} / \mathrm{s}$ bearer is about 80 seconds.

The statistics of the runlengths of the $128 \mathrm{kbit} / \mathrm{s}$ bearer with subsequent upswitching to the $384 \mathrm{kbit} / \mathrm{s}$ bearer is shown in Fig 6. Again, these results are for different mobility scenarios and measured with 'mobile 1'. The runlengths in the 128kbit/s bearer are all $>200$ TTIs which is equal to four seconds in that case. We can observe a jump in the empirical CDFs to $50 \%$ or even $80 \%$ in e.g. the 'walking indoor' scenario. The reason for that jump is the processing time of the switching algorithm together with the decorrelation time of the channel which is obviously shorter than $4 \mathrm{~s}$. Thus, an optimized (faster) switching algorithm could accelerate the switching to the $384 \mathrm{kbit} / \mathrm{s}$ bearer in up to $80 \%$ of the observed cases.

In Fig. 7, we can see that there is no such jump at 200 TTIs in the runlength statistics of the $128 \mathrm{kbit} / \mathrm{s}$ bearer followed by a downswitching to the $64 \mathrm{kbit} / \mathrm{s}$ bearer. The difference in the runlength distributions is the reason for splitting the $128 \mathrm{kbit} / \mathrm{s}$ bearer in two parts for analysis and modeling. In case of the $128 \mathrm{kbit} / \mathrm{s}$ bearer with subsequent downswitching we are able to provide the sum of the statistics of the different mobility scenarios only, due to the fact that for this part of the bearer we do not have sufficient statistics for each of the scenarios. Also in case of the $64 \mathrm{kbit} / \mathrm{s}$ bearer we present the sum of the runlength statistics of all the different mobility scenarios measured with 'mobile 1' (Fig. 8).

The measured runlength distribution for 'mobile 2' and the $384 \mathrm{kbit} / \mathrm{s}$ bearer is shown in Fig. 9. Here again the sum of the measurements of all different mobility scenarios is presented in order to have sufficient statistics.

We can observe that with 'mobile 2' the runlengths of the $384 \mathrm{kbit} / \mathrm{s}$ bearer are $>2 \mathrm{~s}$, and there are runlengths which have a duration of up to 600 s which is much longer than the measured runlengths of 'mobile 1' in the same bearer. The reason for the difference in the runlength distribution between 'mobile 1' and 'mobile 2' is that 'mobile 2' is less sensitive to fading effects and achieves a better service coverage in the network due to a better receiver.

\section{Modeling of DL DCH Dynamic BeARer Type SWITCHING}

\section{A. A Four-state Markov Model}

Based on the measurement results, we first modeled the dynamic bearer switching by a four-state homogeneous Markov chain ( [8], [9]) depicted in Fig. 11, where the valid state transitions are depicted by arrows. The corresponding probability transition matrix $\Pi$ has the following form:

$$
\boldsymbol{\Pi}=\left[\begin{array}{cccc}
\pi_{64,64} & \pi_{64,128 u} & \pi_{64,128 d} & 0 \\
0 & \pi_{128 u, 128 u} & 0 & \pi_{128 u, 384} \\
\pi_{128 d, 64} & 0 & \pi_{128 d, 128 d} & 0 \\
\pi_{384,64} & \pi_{384,128 u} & \pi_{384,128 d} & \pi_{384,384}
\end{array}\right] .
$$

Its elements denote the stationary probabilities $\pi_{i, j}$ of one-step transitions from state $i$ to state $j$. The probability vector $\mathbf{p}$ having the state probabilities $p_{k}$ as elements, can be obtained by solving the system of linear equations $\Pi^{\mathbf{T}} \mathbf{p}=\mathbf{p}$, with constraint $\sum_{k} p_{k}=1$. These probabilities (in our case $\mathbf{p}=$ $\left.\left[p_{384}, p_{128 u}, p_{128 d}, p_{64}\right]\right)$ correspond to the measured values in Fig. 4 (taking here $p_{128 u}$ and $p_{128 d}$ as one state).

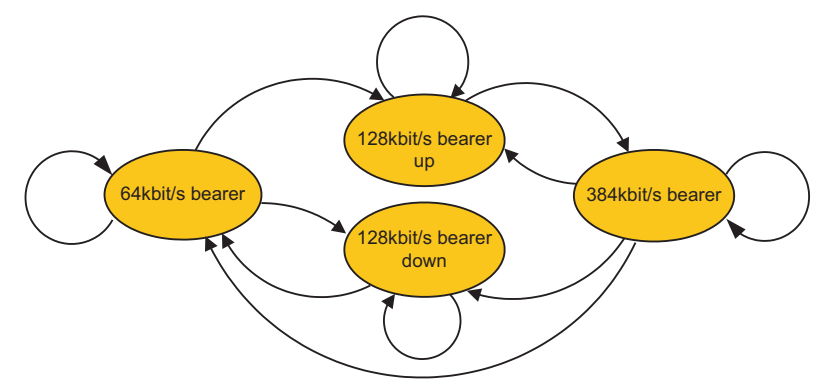

Fig. 11. Schematic illustration of the four-state Markov model for dynamic bearer switching.

As the discretization of time in our discrete-time Markov chain is in TTIs, the jumps to the next states have to be determined every TTI via the corresponding transition probabilities. For the complete description of the model we need eight parameters. Seven parameters are for defining the transition probabilities and one parameter is needed to specify the processing time of the switching algorithm (200 TTIs).

In Figs. 12 to 17 the simulated runlengths (dotted blue line) together with the measured distributions (solid red line) are shown for the different bearers and the two mobiles. In case of Figs. 12 and 13 only the measured statistics of the 'tram 2' mobility scenario are presented as example. It can be observed that the geometric distributions, produced by the Markov chain, are not capable of meeting the measured statistics with sufficient accuracy in some cases - for example the runlength distribution of the $384 \mathrm{kbit} / \mathrm{s}$ bearer of 'mobile 1 ' (Fig. 12). Due to that fact we propose to model the dynamic bearer switching via an alternating Weibull renewal process [10] as shown in the following. 


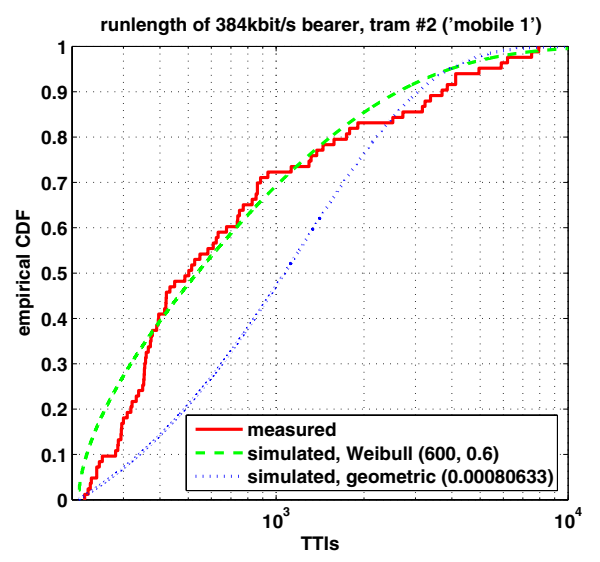

Fig. 12. Runlength distribution of $384 \mathrm{kbit} / \mathrm{s}$ bearer in tramway \#2, measured vs. simulated ('mobile 1').

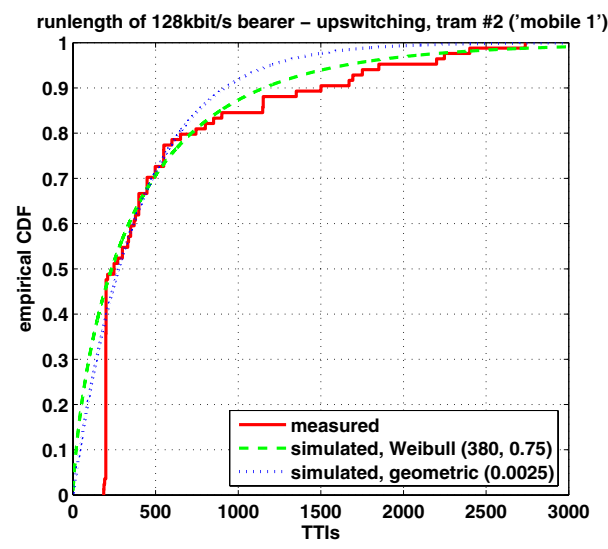

Fig. 13. Runlength distribution of $128 \mathrm{kbit} / \mathrm{s}$ bearer (upswitching) in tramway \#2, measured vs. simulated ('mobile 1').

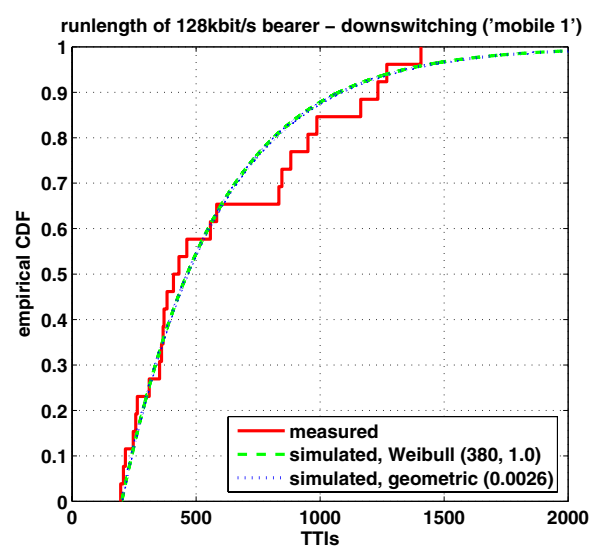

Fig. 14. Runlength distribution of $128 \mathrm{kbit} / \mathrm{s}$ bearer (downswitching), sum of different scenarios, measured vs. simulated ('mobile 1').

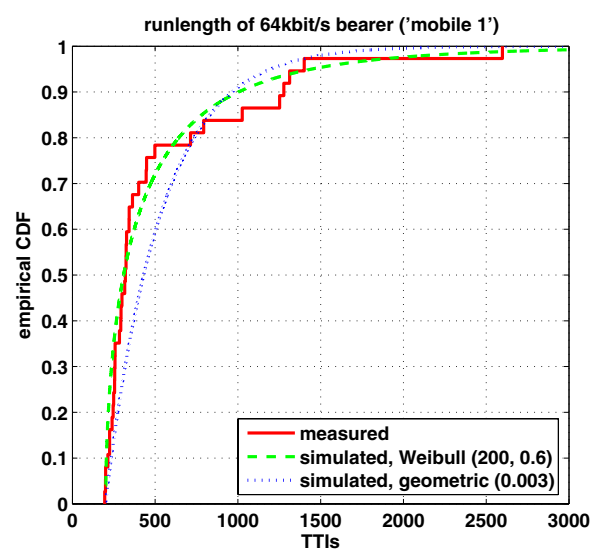

Fig. 15. Runlength distribution of $64 \mathrm{kbit} / \mathrm{s}$ bearer, sum of different scenarios, measured vs. simulated ('mobile 1').

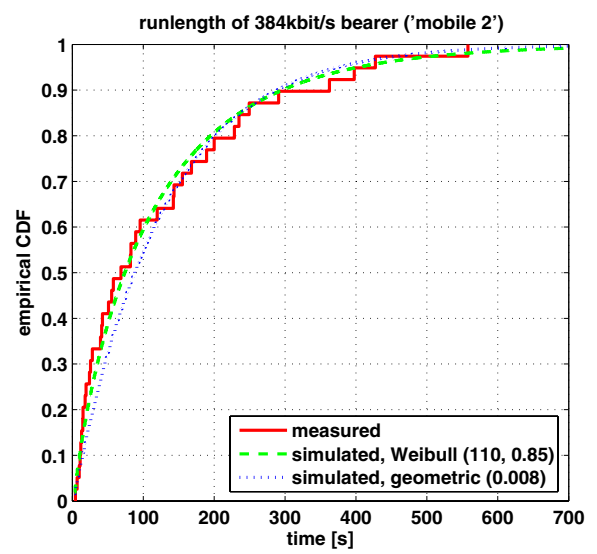

Fig. 16. Runlength distribution of $384 \mathrm{kbit} / \mathrm{s}$ bearer, sum of different scenarios, measured vs. simulated ('mobile 2').

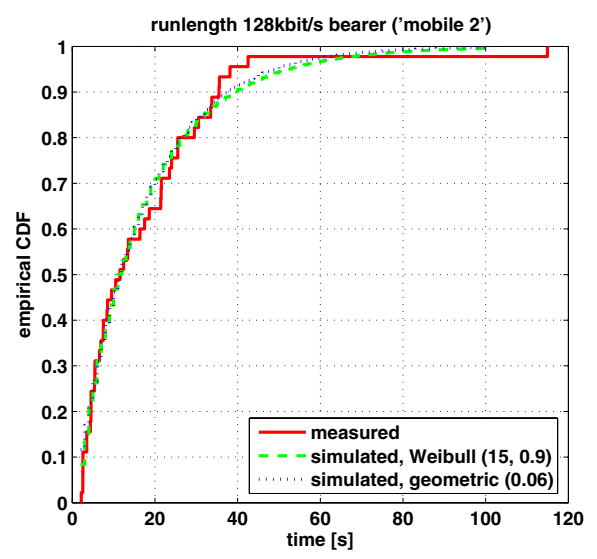

Fig. 17. Runlength distribution of 128kbit/s bearer, sum of different scenarios, measured vs. simulated ('mobile 2'). 


\section{B. Enhanced four-state Model}

In Fig. 12 the empirical cdf of the runlengths of the $384 \mathrm{kbit} / \mathrm{s}$ bearer, measured in a tramway circling the city center of Vienna, is shown. The red solid line shows the measurement result, whereas the dotted blue line shows the simulated result of the Markov model representing a geometric distribution, given by the probability mass function:

$$
\operatorname{pmf}(n)=(1-p)^{n-1} p, \quad n=1,2, \ldots
$$

with parameter $\mathrm{p}$ as extracted out of the measured trace $\left(p=1-\pi_{384,384}\right)$. We can see that the geometric probability distribution is not capable of describing the measured distribution with sufficient accuracy. However, a Weibull distribution of runlengths with the parameters as denoted in the figure meets the measured statistics properly (dashed green line in Fig. 12).

Due to that fact we propose to use a renewal process with four states and Weibull distributed runlengths as an enhanced model for modeling the dynamic bearer type switching for UMTS DL DCH in the PS domain. With the high flexibility of the Weibull distribution we are able to describe all the observed runlength distributions with adequate precision. The two-parameter Weibull cdf is given by

$$
F(x \mid a, b)=1-\exp \left[-(x / a)^{b}\right],
$$

where $a$ and $b$ are scale and shape parameters, respectively.

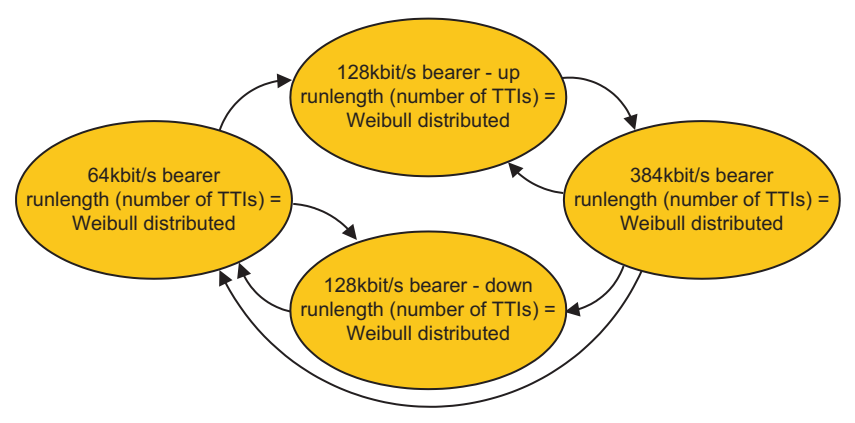

Fig. 18. Schematic illustration of the enhanced four-state Model (alternating Weibull renewal process).

In Fig. 18 a schematic illustration of the model and the allowed jumps between the different states is shown. Such an alternating Weibull renewal process [10] calculates the runlengths in each state via a two-parameter Weibull distributed random number.

The eight parameters for the four standard Weibull distributions (one in each state) together with the three parameters which determine the jumping between the states and one additional parameter for specification of the processing time of the switching algorithm make a total of 12 parameters for complete specification of the model.

Note that the measured distributions of the runlengths and therefore also the model parameters do heavily depend on the network load, the service coverage or the quality of the receiver in the mobile station. If we have for example some congested cells within our route, there will be no or less switching to the $384 \mathrm{kbit} / \mathrm{s}$ or even to the $128 \mathrm{kbit} / \mathrm{s}$ bearer. In case of the varying quality of the receiver (varying service coverage) we have shown that our model is flexible enough to describe the different runlength distributions.

\section{Summary AND CONCLUSIONS}

In this document we present the analysis of dynamic bearer type switching for the UMTS DCH in DL due to measurements in a live UMTS network in the city center of Vienna, Austria. To describe this switching feature offered by UMTS networks we propose a four-state model based on a four-state Markov chain. We show that a Markov chain is not capable of meeting the measured statistics with sufficient accuracy. To overcome this we propose an enhanced model describing a renewal process with Weibull distributed runlengths. We show that with this model the dynamic bearer switching can be simulated properly and the model is flexible enough to be adapted to various mobile receiver qualities or service coverage conditions.

\section{ACKNOWLEDGEMENTS}

We thank mobilkom austria AG\&CoKG for technical and financial support of this work. The views expressed in this paper are those of the authors and do not necessarily reflect the views within mobilkom austria AG\&CoKG.

\section{REFERENCES}

[1] 3GPP TS 25.302 "Services provided by the physical layer," 2005.

[2] H. Holma and A. Toskala, WCDMA for UMTS, Radio Access For Third Generation Mobile Communications, Third Edition, John Wiley \& Sons, Ltd., 2004.

[3] W. Karner, P. Svoboda, M. Rupp, "A UMTS DL DCH Error Model Based on Measurements in Live Networks," in Proc. 12th International Conference on Telecommunications 2005 (ICT 2005), Capetown, South Africa, May 2005.

[4] A. Umbert, P. Diaz, "On the Importance of Error Memory in UMTS Radio Channel Emulation using Hidden Markov Models (HMM)," 15th IEEE International Symposium on Personal, Indoor and Mobile Radio Communications (IEEE PIMRC 2004), vol. 4, pp. 2998-3002, Sept. 2004.

[5] http://www.ericsson.com/products/hp/TEMS_Products_pa.shtml

[6] 3GPP TR 25.993 "Typical examples of Radio Access Bearers (RABs) and Radio Bearers (RBs) supported by Universal Terrestrial Radio Access (UTRA)," 2005.

[7] 3GPP TS 34.108 "Common test environments for User Equipment (UE) Conformance testing," 2005.

[8] A. Papoulis, P.S. Unnikrishna, Probability, random variables, and stochastic processes, McGraw-Hill, 2002.

[9] W. Turin, Digital Transmission Systems: Performance Analysis and modelling. New York: McGraw-Hill, 1999.

[10] D.N.P. Murthy, M. Xie, R. Jiang, Weibull Models, John Wiley \& Sons, Ltd., 2004. 